\section{Allergie als Systemerkrankung}

\author{
Handelt es sich bei Allergie um eine systemische immunologische \\ Reaktion, die, je nachdem an welchem Organsystem sie sich manifes- \\ tiert, zu Asthma, Rhinitis oder Ekzem führt? Diese Frage sollte eine \\ große epidemiologische Studie aus Norwegen klären.
}

Dis ie HUNT-Studie (Nord-Trøndelag Health Study) ist eine bevölkerungsbasierte Studie, in der die Bewohner einer norwegischen Region zu Gesundheitsproblemen, Lebensstil und sozioökonomischen Status befragt wurden. In der Teilstudie Young-HUNT gaben zudem Jugendliche im Alter zwischen 13 und 19 Jahren Auskunft zu Gesundheit und Lebensstil und machten zusätzlich anhand des Fragebogens der International Study of Asthma and Allergy in Childhood (ISAAC) Angaben zu respiratorischen Symptomen, Rhinitis und
Ekzem. Die Daten wurden anschließend mit Hilfe des norwegischen Familienregisters zusammengeführt.

Es konnten die Daten von $8.817 \mathrm{Ju}-$ gendlichen und 5.620 Elternpaaren ausgewertet werden. Bei den Jugendlichen waren Angaben über Wheezing und andere Allergien signifikant assoziiert mit zusätzlichen Gesundheitsproblemen: Die Odds Ratios für die Assoziation von ,nur Wheezing", "Wheezing und Rhinitis" und „Wheezing, Rhinitis und Ekzem“ mit Kopfschmerzen betrugen 2,1, 3,4 und 3,7, mit Muskelschmerzen entspre- chend 2,8, 3,2 und 4,9 und mit Bauchschmerzen 3,6, 4,0 und 4,9. Alle p-Werte waren kleiner 0,01. Ähnliche Resultate ergaben sich, wenn nach allergischem Wheezing gefragt wurde $(\mathrm{p}<0,001)$. Asthma bei den Eltern war assoziiert mit allergischen Symptomen bei den Kindern und elterliches Asthma mit Kopfschmerzen oder Muskelschmerzen korrelierte signifikant mit einer Allergie in Kombination mit ähnlichen Beschwerden bei den Kindern.

Fazit: Allergische Beschwerden bei Jugendlichen sind signifikant mit anderen Symptomen wie Kopf- oder Muskelschmerzen assoziiert und treten familiär gehäuft auf.

$b k$

Tollefsen E et al. Allergy: a systemic disease? The HUNT and Young-HUNT study, Norway. Pediatr Allergy Immunol 2008; 19: $730-6$

\title{
Hyposensibilisierung hilft bei Otitis
}

\section{Neben Rhinitis, Asthma oder Ekzem wird auch das Auftreten der chronischen Otitis media im Rahmen einer Atopie diskutiert. Ein HNO-Arzt aus Boston, USA, liefert dazu jetzt interessante Daten.}

ber die Genese der chronischen Otitis media besteht bis heute wenig Klarheit. Lange Zeit nahm man als Ursache anatomische oder funktionelle Störungen der Eustachischen Röhre an - eine These, die mittlerweile im Widerspruch zu vielen Publikationen steht. Einige Untersuchungen konnten belegen, dass bei Patienten mit Otitis media eine TH2-dominierte Immunreaktion im Sinne einer allergischen Erkrankung vorliegt.

Vor diesem Hintergrund wurden in einer HNO-Praxis 89 Kinder und Erwachsene untersucht. Alle Patienten litten an einer chronischen Otitis media mit Erguss, bei der entweder eine Parazentese mit Einlegen von Paukenröhrchen anstand oder bei der eine chronische Sekretion aus einem Trommelfell nachweisbar war. Alle Patienten unterzogen sich einer Allergietestung, wonach eine spezifische Immuntherapie

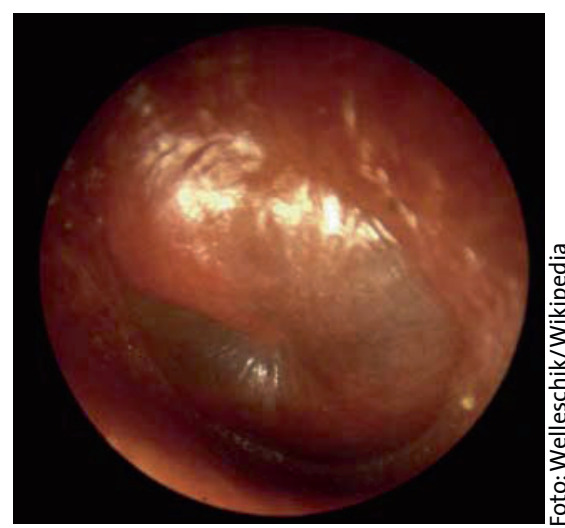

Eine Indikation für eine spezifische Immuntherapie?

eingeleitet wurde. Als Kontrolle diente eine Gruppe von 21 Patienten, die die Therapie abgelehnt hattten. Die Teilnehmer wurden dann bis zu acht Jahren nachbeobachtet.

Die meisten Patienten waren allergisch auf Hausstaub (94\%), Schimmel- pilze (88\%) und Tierhaare (44\%), 9\% waren allergisch auf saisonale Pollen. Bei der überwiegenden Zahl der Studienteilnehmer lag eine Polysensibilisierung vor. An Otitis media als einziger Erkrankung litten nur 37\% der Patienten. Häufig trat die Otitis mit Rhinitis/Sinusitis (63\%) und Asthma (21\%) zusammen auf.

Nach der Immuntherapie waren $85 \%$ der insgesamt 127 entzündeten Mittelohrregionen frei von Erguss bzw. Sekretion. Nur bei acht Patienten zeigte sich im Studienverlauf keine Remission. In der Kontrollgruppe ohne Therapie trat bei keinem der Studienteilnehmer eine Besserung der Otitis-media-Symptomatik auf.

Fazit: Nach den Ergebnissen dieser Studie scheint die chronische Otitis media zu den Erkrankungen des atopischen Formenkreises zu gehören: Alle Patienten wiesen Allergien auf, in $85 \%$ der Fälle führte die Hyposensibilisierung zu einer Heilung.

Hurst DS et al. Efficacy of allergy immunotherapy as a treatment for patients with chronic otitis media with effusion. Int J Pediatr Otolaryngol 2008; 72: 1215-23 